NASA Technical Memorandum 79730

Standard Techniques

for Presentation and Analysis

of Crater Size-Frequency Data

APRIL 19:8

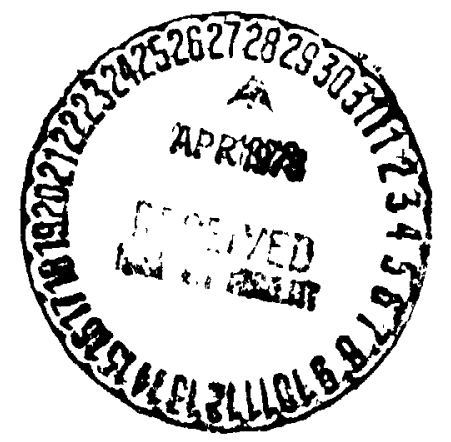


NASA Technical Memorandum 79730

\section{Standard Techniques \\ for Presentation and Analysis \\ of Crater Size-Frequency Data}

Crater Analysis Techniques Working Group

Office of Space Sciezce

Ir'ashington, D.C. 
Crater Analysis Techniques

Working Group

\begin{abstract}
(Alphabetically) R. Arvidson, Washington, Univ.; J. Boyce (Cochairperson), USGS/NASA Hdqt; C. Chapman, Planetary Sciences Inst.; M. Cintala, Brown Univ.; M. Fulchignoni, Lab. Astrofiscia Spacial CNR; H. Moore, USGS; G. Neukum, Univ. Munich; P. Schultz, Lunar and Planetary Inst.; L. Soderblom, USGS; R. Strom, Univ. Arizona; A. Woronow (Chairperson), Univ. Arizona; R. Young, SUNY.
\end{abstract}


TABLE OF CONTENTS

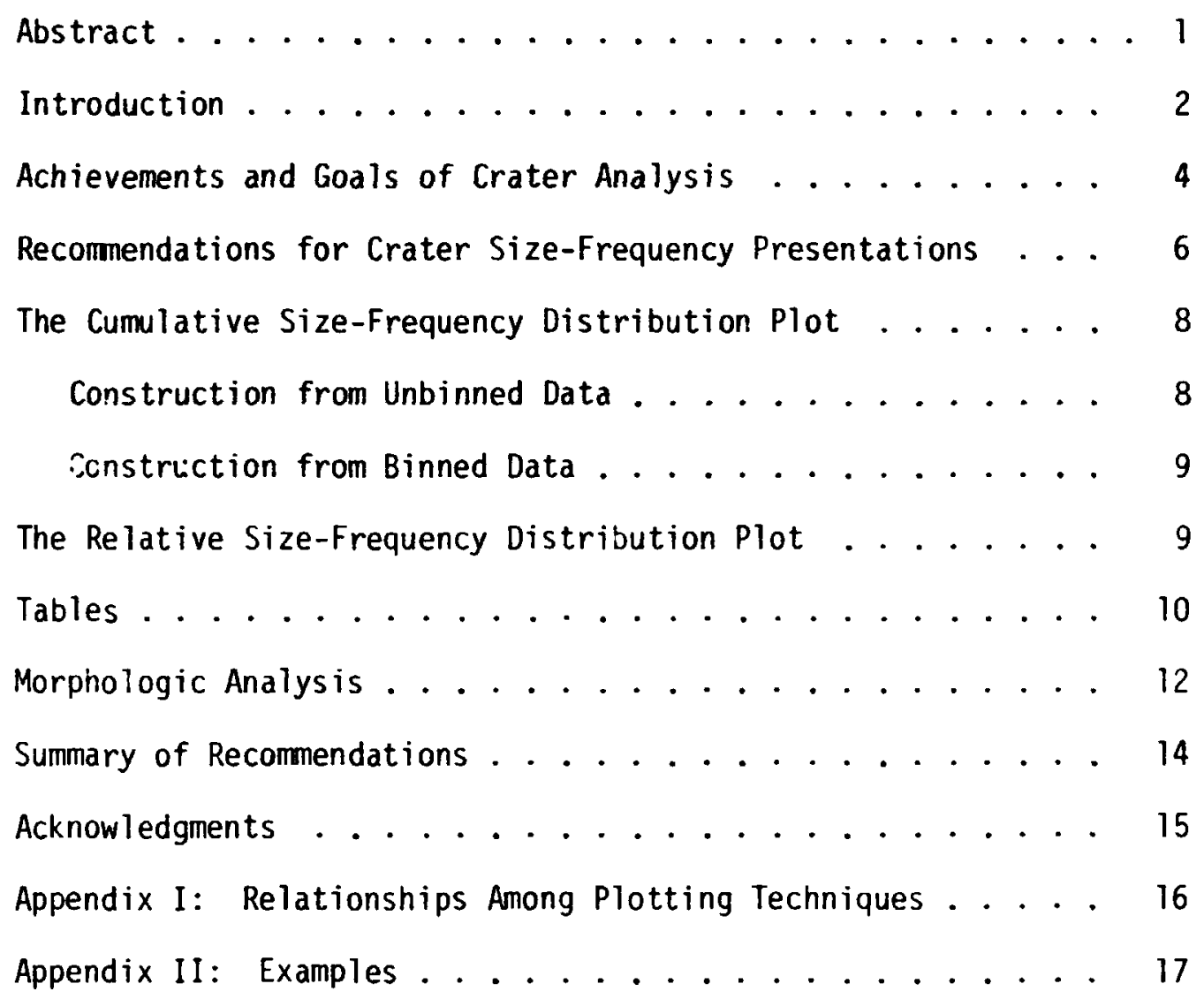

Illustrations

Figure 1. Example of a "Relative Size-Frequency Distribution Plot"..................... 19

Figure 2. Example of a "Cumulative Size-Frequency Distributron Plot"............... 20

Tables

Table 1. Lunar Mare: Frontside Post Mare (data set 'a') . 18

Table 2: Mare Crisium: Eastem Part (data set 'b') . . . . 18 


\section{ABSTRACT}

In September 1977, a crater studies workshcp was held for the purpose of developing standardized data analysis and presentation techniques. This report contains the unanimous recommendations of the participants. This first meeting considered primarily crater size-frequency data. Future meetings will treat other aspects of crater studies such as morphologies. 


\section{INTRODUCT ION}

Analyses of crater population statistics have played a major role in establishing planetary chronologies, in determining the nature of planetary surface processes, and in inferrring the characteristics of asteroidal and cometary populations and the meteoroid complex. These analyses also have been a reliable tool for probing the physical environment of planetary surfaces and interiors. The wide array of applications of crater analyses has brought investigators of diverse interests and backgrounds into this relatively young discipline; and a myriad of data analysis techniques have arisen. Most of the techniques are fundamentally the same, and attempt to display or analyze the same attributes of the crater populations, but the final products have markedly different appearances and often are not easily intercompared. By standardizing analys is techniques, redundant data collection and reduction will be mininized and, most importantly, underlying concepts can be traced more easily through related studies. Toward the soal of standardization, a working group was formed. The group met September 8 and 9, 1977, at the USGS facility in Flagstaff, $A z$. This document contains the findings and recommendations resulting from that meeting.

Because "crater analyses" involve such a broad range of disciplines, the working group chose to concentrate on crater size-freojency analysis at its first meeting, with other topics left until future meetings. The group members represent a cross-section of institutions and interests, but all have in common current involvement in crater size-frequency data collection and reduction. A draft of this report has been widely circulated for comment to an extensive list of scientists whose research incorporates 
crater analysis. Those scientists responding are listed in the acknowledgments and their comments have been taken into account in this final report.

In this document italics emphasize the specific recomrandations of the committee. All of the recomendations presented were adopted unanimously by the committee.

Before presenting the individual recommendations, a brief review of the past accomplishments and current topics of investigation utilizing crater size-frequency analysis will establish a perspective from which to view the body of this report. 


\section{ACHIEVEMENTS AND GOALS OF CRATER ANALYSIS:}

Crater statistical analyses have been used successfully in three major ways, namely: (1) to understand planetary chronologies and to date geologic units and events, (2) to identify surface and subsurface processes, and (3) to provide a framework for interpretation of data from other fields.

Dating of planetary surfaces was one of the first applications of crater statistics. Through detailed study of crater size-frequency, density, and morphology distributions, reliable relative chronologies are being established for the Moon, Mars, and Mercury. For the Moon, where Apollo and Luna returned samples are available, Moon-wide absolute chronologies have been developed and are being extended across the surface. Many sub-disciplines of planetology require at least a framework of relative ages in order to interpret planetary evolution in terms of chemistry, structure, and processes; crater analyses are the chief means of obtaining this time framework. When combined with asteroid and comet surveys, crater statistics provide the only presently available means of estimating absolute (admittedly coarse) chronologies for Mercury, Venus, and Mars.

Crater analyses have established the generally ancient nature of the surfaces of the Moon, Mercury, and Mars, thereby demonstrating the uniqueness of the Earth's rapid tectonic cycle of crustal production, "'slution, and destruction. Lunar crater statistics and returned sampic ages are combined to establish that the impact flux in the Earth-Moon system has been highly time-dependent with an early high flux that dropped rapidly to a level several orders of magnitude lower with possible episodic excursion back to higher levels. 
Analys is of microcrater populations on exposed lunar rocks has yielded exposure time information and has helped to establish rates for such processes as lunar rock erosion, regolith mixing, solar flare irongroup particle track formation, solar wind sputtering, and accumulation of solar wind elements.

Progress continues to be made in establishing both relative and absolute chronologies and in improving impact-flux histories. We believe that crater analyses will provide improved absolute dates for the surfaces of all the terrestrial planets and better interplanetary correlations of geologic time.

Because impact craters form at random locations on planetary surfaces, but geologic events often locally alter this uniformity, crater analyses have been useful for mapping geologic units of common origin and age. Furthermore, different geologic processes (e.g. aeolian erosion, volcanism, and tectonic disruption) affect crater size-frequency distributions and morpinologies in characteristic manners. Through crater analyses a wide range of internal and surface geologic processes have been identified or contrasted on the Moon, Mercury, and Mars.

Other areas of planetology utilized knowledge established by cratering mechanics and statistical studies in order to interpret their own observations. Among the many possible examples are correlation of eclipse temperatures with surface impact ages; correlation of bistatic-radar echo broadening with crater densities and size distribution; interpretation of the $25 \mathrm{~km}$-deep change in lunar p-wave velocity in terms of crustal fracturing from impact events; and correlation of lunar reflection spectra with impact-glass content. 
Crater analyses already have produced valuable and otherwise unattainable information, and the prospects for still further successes are excellent. Current competition and debate are producing rapid advancements and refinements of our understanding of cratering as a geologic process; recognition of the characteristics of primary and secondary cratering; understanding of the origin, evolution, and dynamics of small bodies in the solar system; and understanding of planetary crustal properties, environments, and evolution. As the techniques of crater analysis continues to expand the library of basic information about the terrestrial planets, their use and respect by other disciplines will continue to increase.

RECOMMENDATIONS FOR CRATER SIZE-FREQUENCY PRESENTATIONS:

A wide variety of presentation techniques for crater data have appeared in the literature. Occasionally the data appear in tables, more often in graphs. Although each of the techniques has its particular strengths and weaknesses, all fall into two broad classes: those that display the differential size-frequency distribution and those that display its integral, the cumulative size-frequency distribution. The diversity of graphic technıques in use has made communication among researchers difficult. For this reason we have developed a system of standardization of crater statistics techniques.

The singularly most important benefit derived from standardized crater analysis techniques is enhanced communication. Another benefit is minimization of redundant measurements. Plotting data in standard formats will perrit rapid comparisons among individual studies and data sets. In particular, related features of the data will be at the same location 
( $x$ and $y$ values) and look the same on all plots. Oral communication will also benefit because reference to a bump or wiggle at some set of coordinates will unambiguously locate that feature.

The criteria listed below established the basis for selecting the two plotting techniques. The technique(s) should: (1) be in common use, or easily understood; (2) be easily implemented; (3) be readily interpreted; (4) have wide applicability to recognized problems so that the need to use alternative techniques is minimized; and (5) be both "consistent" and "unbiased" (in the statistical sense) and insensitive to the data gathering techniques. The plot should not depend strongly on the craterdiameter bin size used.

Based on the above criteria, we chose two plotting techniques as the standards; the "Cumulative Size-Frequency Distribution Plot" and the "Relative Size-Frequency Distribution Plot". The former possesses the attributes and benefits of a cumulative plotting technique, while the latter is a variation on a differential plotting technique. We recommend that all auta be plotted in both of the standard formats. However, we recognize that all desirable crater analyses may not be served by just these two plots. In such cases, special needs take priority over the recommended standard formats, but the standard formats should still be presented, in addition to the special formats. This will enable others easily to utilize the data sets.

Details of these plotting techniques are given below. First however, we recommend using the following basic conventions of plotting: (1) Use double logarithmic axes of the saile scale. (2) Both axes should have? consistent units. That 15 ; if crater diameters were given in kilometers, 
crater densities should be in number per square kilometer. (3) Pzot one standard deviation, 10, confidence interuals for each data point plotted. For most purposes this can be easily done by assuming that the populations are Poisson distributed then $l_{\sigma}$ can be estimated by using $N= \pm \sqrt{N}$ where $N$ is the number of craters.

THE CUMULATIVE SIZE-FREQUENCY DISTRIBUTION PLOT:

The Cumulative Size-Frequency Distribution Plot possessus a number of important attributes:

(1) It displays the integral of $F(D)$.

(2) It is a well-known and easily implemented technique.

(3) The plotted results are fairly independent of the bin size (but resolution is not independent of bin size).

Construction from Unbinned Data: When the diameter of individual craters are measured and recorded, the data are ungrouped or unbinned, and when crater diameters are measured and recorded by keeping a tally of the number of craters in increments the data are grouped or binned. Unbinned data provides the greatest flexibility because they can easily be recast into binned data and they provide the maximum possible resolution of small features in the distribution function. However, collection, manipulation, and display 0:: unbinned data is more time consuming than for binned data. The required steps for plotting unbinned data are:

(1) Sort the craters into descending order by diameter (d); obtaining the sequence $d_{1}, d_{2}, d_{3}, \ldots d_{n}$.

(2) Plot the $\log$ of the diameter of each crater $d_{n}, \log \left(d_{n}\right)$, against the $\log$ of the total or cumulative number of craters per unit area with equal or larger diameters, $\log (n / A)$, where $A$ is the surface area. 
(3) Plot the confidence interval $\pm \sigma$, which for the nth crater is $\log \left(\frac{n \pm \sqrt{n}}{A}\right)$.

Construction from Binned Data: For many purposes the crater diameter measurements can be taken in bins or be binned before plotting. The widths of the bins can be adjusted to achieve the desired level of resolution. Let the largest diameter bin be bin 1 containing $n_{1}$ craters $\left(n_{1} \geq 1\right)$, and the second largest bin be bin 2 containing $n_{2}$ craters ..., and the ith diameter be bin $i$ containing $n_{i}$ craters. To plot the data in bin $i$ which has diameter limits $D_{a}$ to $D_{b}\left(D_{b}>D_{a}\right)$ and contains $n_{i}$ craters, plot $\log (\rho)$ where, $\rho=\sum_{K=1}^{i} \frac{n_{K}}{A_{K}}$, against $\log \left(D_{a}\right)$. This equation allows the population in each bin to have been measured on a different amount of surface area. Letting $N=\sum_{K=1}^{i} n_{K}$, the total number of craters in bin $i$ and all larger diameter bins, the confidence interval, $\pm \sigma$, is $\log \left(\rho \pm \rho \frac{1}{\sqrt{N}}\right)$.

THE RELATIVE SIZE-FREQUENCY DISTRIBUTION PLOT:

The Relative Size-Frequency Distribution Plot possesses a number of important attributes:

(1) It is easily implemented.

(2) The plot displays information on the differential size-frequency distribution function $F(D)$.

(3) The plotted results are as independent of the bin size as possible (but resolution is not independent of the bin size).

(4) Most crater populations have slope indices within the range of \pm 1 of the function $D^{-3}$; therefore, they will plot as non-sloping or moderately sloping lines on these plots. The shallow average slopes of lines on these plots make any changes in the $F(D)$ more obvious and facilitates identifying differences in distribution functions and densities among crater populations. 
Prior to plotting the data by this technique, they must be binned. Consider a bin with diameter limits $D_{a}$ to $D_{b}\left(D_{b}>D_{a}\right)$ containing $n$ craters which were measured on a surface of area $A$. To determine the value to be plotted along th absicissas, first calculate the geometric mean (D) of the craters in the bin. If the individual diameters (d's) are available, $\bar{D}=\left(\left.\begin{array}{ll}n & \\ \prod_{j=1} & d_{j}\end{array}\right|^{1 / n}\right.$. the $n$th root of the product of the diameters. If the individual diameters are not available, $\bar{D} \approx \sqrt{D_{a} D_{b}}$. To plot the data in bin $i$, plot $\log (R)$ where $R=(\bar{D})^{3} n / A\left(D_{b}-D_{a}\right)$ against $\log (D)$. The confidence interval, \pm 0 , is $\log \left(R \pm \frac{R}{\sqrt{N}}\right)$.

\section{TABLES:}

Ali data should be given in tabular form. Exceptions to this are data published in a previous article or data from a widely accessible crater catalog. Some data may be so exiensive that giving the full set of tables in an article would result in excessive expense or article length. In this case, the author should use the journal's microfiche service, if available. Whether or not this service is available, a reprint of all articles and their $\therefore a^{+}$i tai $i$ s shouid be sent to the Lunar and Plonetary Institute ( $E F I$ ) 3303 Whir Boad 1, Hulezun, Texas, 77058, for arciniving. If the data are not presented in the article then state where the tabulated data can be obtained. Thus, data sets will be available to others for further analysis and redunciant crater measurements will be minimized.

In order to maximize the data's usefuilness, the tailea should comply Witi $x$ standard format. We are aware that some data collection techniques make strict compliance to the following format impossible. However, tables should comply with as many of the following rules as possible. The standard format is: 
(1) All data should be binned for the tabular presentation, regardless of how they were taken or plotted. The bins should have geometric widths of not greater than $\sqrt{2}$. A bin boundary, or projected boundary, should be at $D=1 \mathrm{~km}$ (e.g. bins of $1 / \sqrt{2}$ to $1 \mathrm{~km}, 1$ to $\sqrt{2} \mathrm{~km}, \sqrt{2}$ to $2 \mathrm{~km}$ satisfy this format). If finer bins are desirable, using roots of $\sqrt{2}$ will allow combining that data with other data binned as described $\bar{r}$ ove.

(2) The number of craters within each bin should be given. Do not give the cumulative number or the number per unit area.

(3) Specify the area of the surface over which the measurements were made. If the area changes from bin to bin, give it for each bin.

Supporting information for each table must also be given. It should include, but not be limited to, the following:

(1) A description of the type of materials from which the measurements were made. Give photograph I. D. number, type of processing it received, the scale used to convert the measurements to the actual diameter, the source of the scale, and whether the scale was assumed to be constant over the entire photograph.

(2) Give the exact location of the region studied. Either latitudelongitude limits or a location chart can be used.

(3) Specify the method of data collection and reduction. Describe the measuring device (ruler, particle-size analyzer, etc.) and its accuracy. Describe all corrections made to the measurements (such as for sun angle) and why those corrections were needed.

(4) Give all other data that wouid be necessary for the reader to be able to reconstruct the data set from the appropriate photographs. 
Regression analyses: If the data are regressed to a straight line, a power series, a specific modez, or other function, al indication of the success of the regression should be given. In most cases this is best done by using a non-parametric test (e.g. $\chi^{2}$ or Kilmogoroff-Smirnoff test) to compare the data and the regression results. A test insures that the regression adequately represents the data. In addition to a goodness-offit test, the lo confidence interval on the regression coefficients should be given. Non-parametric tests are also useful for examining the range of admissible models.

MORPHOLOGIC ANALYSES:

The committee deferred extensive formulation of standards for crater morphology studies until a later meeting. Nonetheless, morphologic studies cannot be considered completely separate from statistical analyses, and the committee decided to lay the foundation for future discussions with the following two recommendations:

(1) A quantitative measurement is always to be preferred over a qualitative one. Assignments of morphologic characteristics to subjective classes (whether the classes are numbered 1, 2, 3, or called high, medium, low, etc.) do not form a unique basis for statistical evaluation of the conclusions. Quantitative measurements (whether from a continuum such as inside/outside diameter ratios, or a Boolean true-false evaluation such as central peak presence or absence) can be unambiguously treated and are less subject to alternative interpretation resulting from differences in subjective judgments. Therefore, if a hypothes is could be tested by either a quantitative or qualitative morphology measurement, the quantitative measurement is to be preferred. 
(2) In many instances a quantitative measurement may not be possible. If a qualitative measurement is necessamy, the exact criteria for making the qualitative judgments must be given. A detailed description of the criteria may suffice, but specific examples illustrating applications of the criteria would be preferable. 


\section{SUMMARY OF RECOMMENDATIONS}

Graphs:

1. Display data on both a "Cumulative Size-Frequency Plot" and a "Relative Size-Frequency Plot".

2. Make the abscissas and ordinates both base 10 logarithmic.

3. Use consistent units on both axes.

4. Use the same scale for both axes.

5. Plot the lo confidence intervals for the data points.

Tables:

1. Give all the data in tabular form.

2. Bin the data in $\sqrt{2}$-factor or finer bins.

3. Give the number of craters in each bin.

4. Specify the amount of surface area on which the data were measured.

5. Deposit the data tables with LPI.

Supporting Information:

1. Specify the source and kind of materials on which the measurements were made.

2. Specify the measuring technique used and assess its accuracy.

3. Give the exact location of the area studied.

4. Give all other pertinent information such as the assumed scale of the photographs and the corrections, if any, made to the raw measurements.

Regression Analyses:

1. Give the $1 \sigma$ confidence intervals on the regression coefficients.

2. Report the results of a goodness-of-fit test of the data to the regression.

Morphologic Analyses:

1. A quantitative measure is preferable to a qualitative measure.

2. Specify the exact criteria used for making qualitative assignments or measurements. 


\section{ACKNOWLEDGMENTS}

We wish to thank the following individuals for reviews and suggestions:

C. C. Allen, R. B. Baldwin, K. R. Blasius, B. M. Cordell, T. L. Duxbury, D. E. Gault, W. K. Hartmann, J. B. Hartung, B. R. Hawke, J. W. Head, F. Hörz, L. D. Jolle, K. C. Jones, M. Leake, G. E. McGill, R. J. Pike, J. Roberts, E. A. Whitaker. We also thank the NASA Planetary Geology Programs Office for their support and encouragement. 


\section{APPENDIX I \\ Relationships Among Plotting Techniques}

Basic definitions:

(1) Surface area is $A$.

(2) Let $F(D)$ be the differential size number distribution of the craters per unit area.

(3) $C(D)$, the cumulative number of craters with diameters greater than or equal to $D$ and less than or equal to $D_{\max }$ (the largesi

crater measured) per unit area is $C(D)=\frac{1}{A} \int_{D_{\max }}^{D} F(D) d D$.

Each plotting technique will be related to $F(D)$ or $C(D)$.

I. Cumulative Size-Frequency Distribution Plot:

- This is given by definition (3). We plot $\log (C(D))$ against $\log (D)$.

II. Relative Size-Frequency Distribution Plot:

- Let $S(D)$ be a differential "reference distribution":

$S(D) \equiv D^{-3}$

- $R(D) \equiv \frac{F(D)}{S(D)}$. To apply this definition we evaluate $R(D)$ over the finite interval $\Delta D$ between $D_{a}$ and $D_{b}\left(D_{b}>D_{a}\right)$ :

$$
R(D) \approx \frac{\Delta N}{\Delta D} \frac{1}{S(D) A}=\frac{C\left(D_{a}\right)-C\left(D_{b}\right)}{\left(D_{b}-D_{a}\right) S(D)}
$$

- $S(D)$ is evaluated at the geometric mean of the interval $D_{a}$ to $D_{b}$ : $S(D)=\left(D_{a} D_{b}\right)^{-3 / 2}$.

- Therefore,

$$
R(D) \approx\left(D_{a} D_{b}\right)^{3 / 2}\left(\frac{C\left(D_{a}\right)-C\left(D_{b}\right)}{D_{b}-D_{a}}\right)
$$

- We plot $\log (R(D))$ against $\log \left(\left(D_{a} D_{b}\right)^{\frac{1}{2}}\right)$. 
III. Differential Size-Frequency Distribution Plot (e.g. Chapman and Haefner, JGR, 1967):

- This method approximates $F(D)$ over the interval $D_{a}$ to $D_{b}$

$$
\begin{aligned}
& \left(D_{b}>D_{a}\right): \\
& \quad F(D) \approx \frac{C\left(D_{a}\right)-C\left(D_{b}\right)}{D_{b}-D_{a}}
\end{aligned}
$$

- We would plot log $(F(D))$ against $\log \left(\frac{D_{b}-D_{a}}{2}\right)$

IV. Incremental Number Plot (e.g. Hartmann, Comm., Lunar and Planetary Laboratory, 1964):

$-J(D)=C\left(D_{a}\right)-C\left(I D_{a}\right)$

Where $I$ is a constant factor greater than unity (often $I=\sqrt{2}$ )

which must be maintained for the entire span of the plot.

- We would plot $\log (J(D))$ against $\log \left(D_{a} I^{\frac{1}{2}}\right)$.

V. Area Plot (e.g. Strom, Abs. 8th LSC, 1977):

- $T(D)=\frac{\pi(\bar{D})^{2}}{4} J(D)$

- Where $D=D_{a} I^{\frac{3}{2}}$ ( $I$ is as defined in IV above).

- Therefore, $T(D)=\frac{\pi D_{a} I^{\frac{1}{2}}}{4}\left(C\left(D_{a}\right)-C\left(D_{b}\right)\right)$.

- We would plot $\log (T(D))$ against $\log \left(D_{a} I^{\frac{1}{2}}\right)$.

\section{APPENDIX II - EXAMPLES}

Below are given two sets of crater size-frequency data in tabular and both graphic formats. Table I is data from the new LPL lunar catalog. This table conforms exactly to the suggested format: $\sqrt{2}$ geometric bins with a projected bin boundary of $1 \mathrm{~km}$ diameter. These data are labeled a in Figures 1 and 2. The second table is rebinned data from Neukum et al. (Moon, 1975). The bins have not been made into $\sqrt{2}$ intervals, but a bin boundary is at $\mathrm{l} \mathrm{km}$ diameter. This data is labeled $\underline{b}$ in Figures 1 and 2 . 


\section{TABLE I}

Lunar Mare: Frontside Post Mare (data set 'a')

\begin{tabular}{rcc}
$\begin{array}{c}\text { Bin Diameter } \\
(\mathrm{km})\end{array}$ & $\begin{array}{c}\text { Number of Craters } \\
\text { per Bin }\end{array}$ & $\begin{array}{c}\text { Surface Area } \\
(\mathrm{km})\end{array}$ \\
\hline $8.0-11.3$ & 91 & $6.67 \times 10^{6}$ \\
$11.3-16.0$ & 60 & $6.67 \times 10^{6}$ \\
$16.0-22.6$ & 22 & $6.67 \times 10^{6}$ \\
$22.6-32.0$ & 19 & $6.67 \times 10^{6}$ \\
$32.0-45.3$ & 7 & $6.67 \times 10^{6}$ \\
$45.3-64.0$ & 6 & $6.67 \times 10^{6}$ \\
$64.0-90.5$ & 2 & $6.67 \times 10^{6}$ \\
$90.5-128.0$ & 2 & $6.67 \times 10^{6}$ \\
$128.0-181.0$ & 1 & $6.67 \times 10^{6}$
\end{tabular}

TABLE II

Mare Crisium - Eastern Part (data set 'b')

\begin{tabular}{ccc}
$\begin{array}{c}\text { Bin Diameters } \\
(\mathrm{km})\end{array}$ & $\begin{array}{c}\text { Number of Craters } \\
\text { per Bin }\end{array}$ & $\begin{array}{c}\text { Surface Area } \\
\left(\mathrm{km}^{2}\right)\end{array}$ \\
\hline $0.8-0.9$ & 85 & $4.4109 \times 10^{4}$ \\
$0.9-1.0$ & 49 & $4.4109 \times 10^{4}$ \\
$1.0-1.2$ & 57 & $4.4109 \times 10^{4}$ \\
$1.2-1.4$ & 25 & $4.4109 \times 10^{4}$ \\
$1.4-1.8$ & 15 & $4.4109 \times 10^{4}$ \\
$1.8-2.2$ & 11 & $4.4109 \times 10^{4}$ \\
$2.2-3.6$ & 10 & $4.4109 \times 10^{4}$ \\
$>3.6$ & 1 & $4.4109 \times 10^{4}$
\end{tabular}




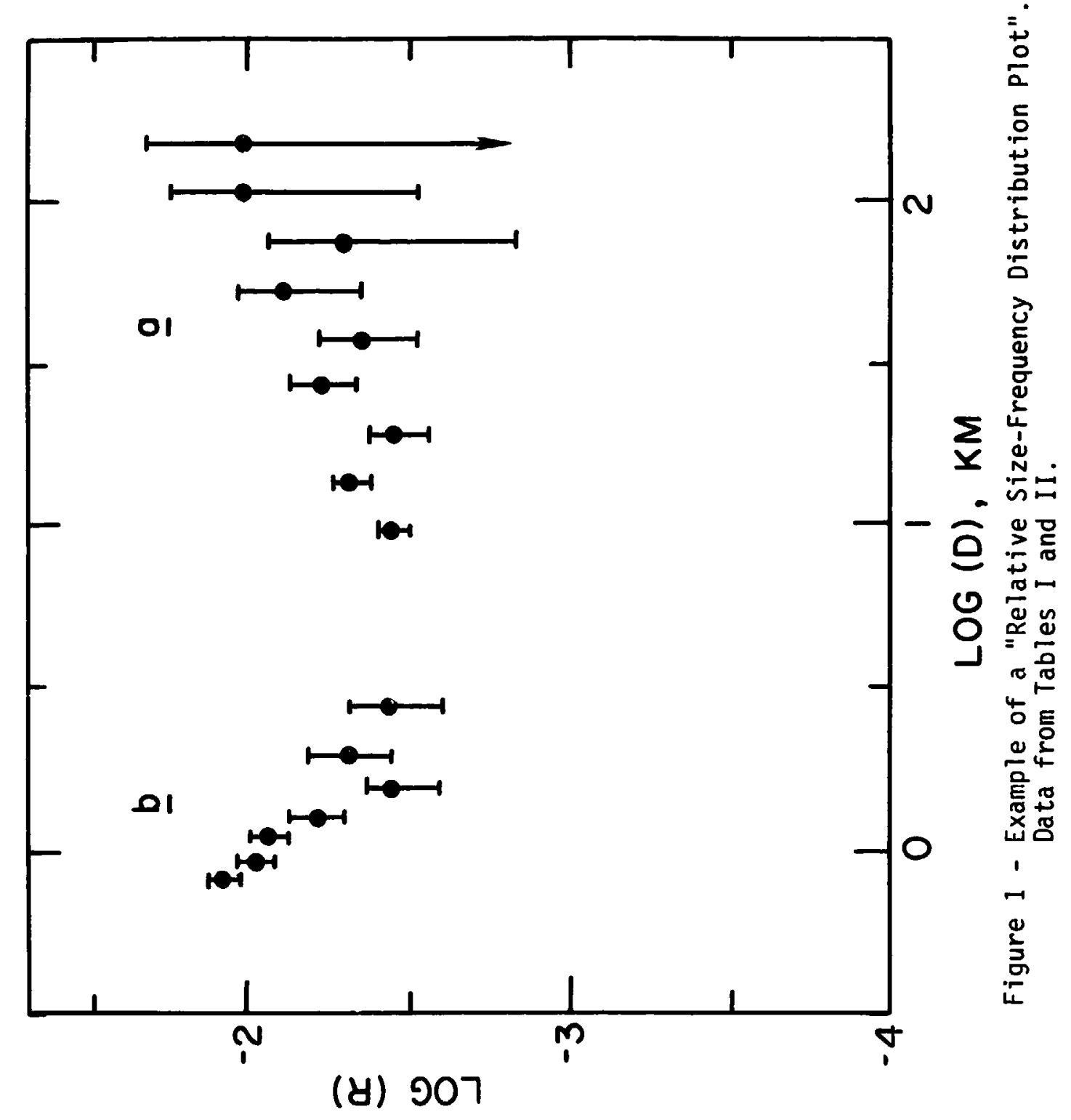




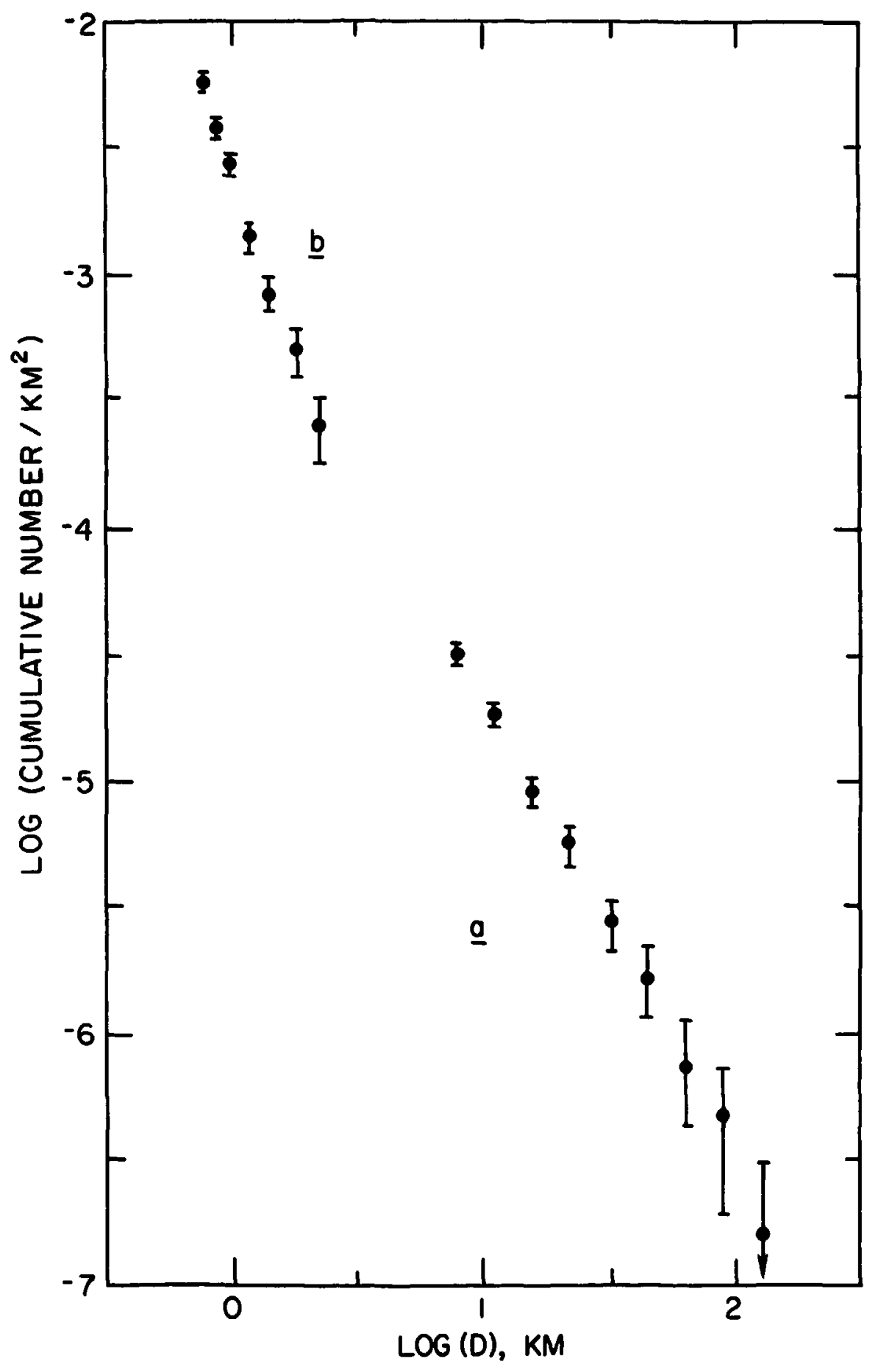

Figure 2 - Example of a "Cumulative Size-Frequency Distribution Plot". Data from Tables I and II. 


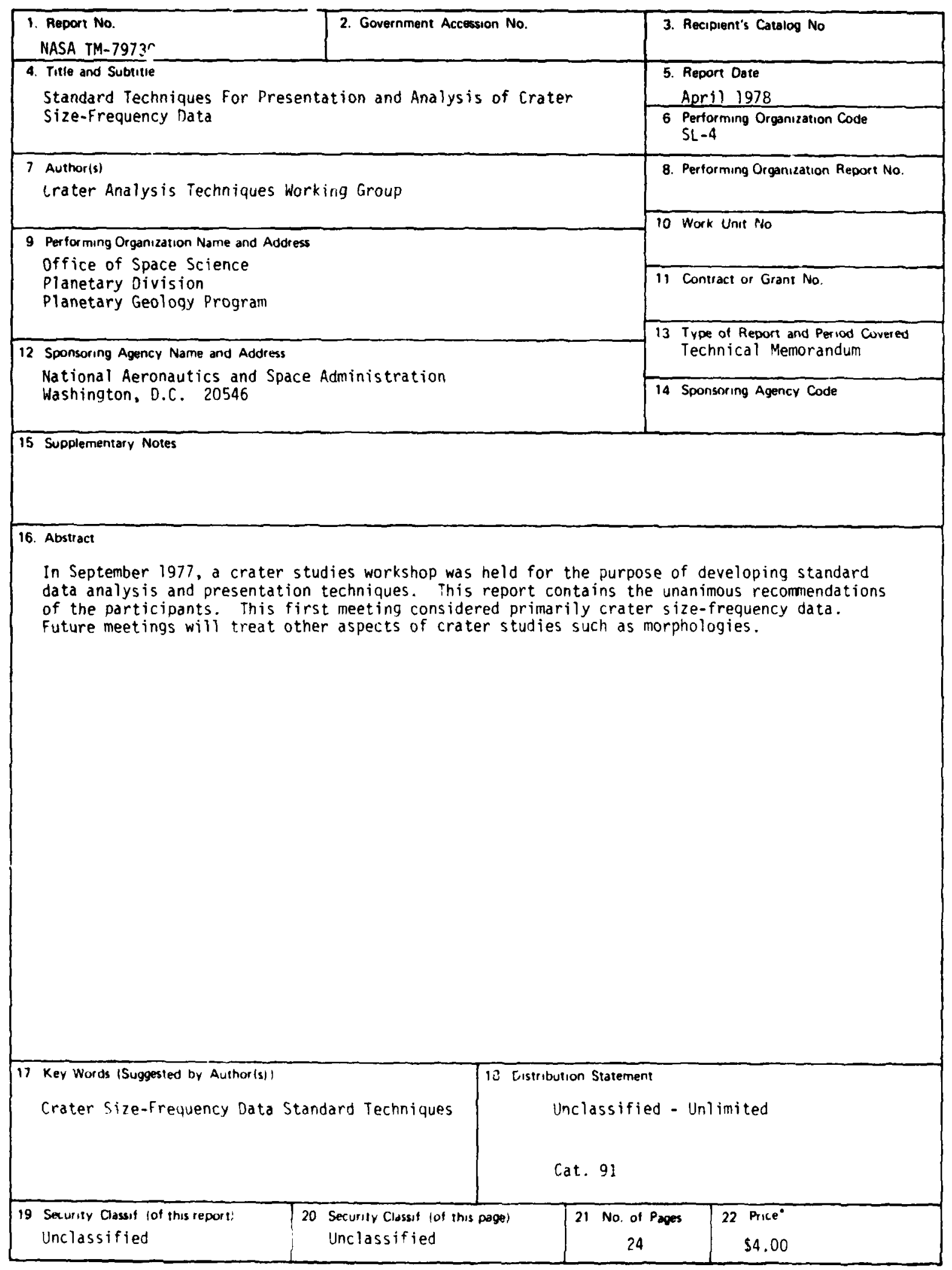

For sale by the Nerional Technical Information Service, Springfield, Virginia 22161 